IFAC Proceedings Volumes (IFAC-PapersOnline), 18(1), 2011, pp. 2494-2505 (doi:10.3182/20110828-6-IT-1002.03315) (survey lecture)

\title{
Analytical-numerical methods for investigation of hidden oscillations in nonlinear control systems
}

Leonov G.A., Kuznetsov N.V.

Draft $^{1} 23$

\begin{abstract}
The method of harmonic linearization, numerical methods, and the applied bifurcation theory together discover new opportunities for analysis of oscillations of control systems. In the present survey analytical-numerical algorithms for hidden oscillation localization are discussed. Examples of hidden attractor localization in Chua's circuit and counterexamples construction to Aizerman's conjecture and Kalman's conjecture are considered.
\end{abstract}

Keywords: hidden oscillation, hidden attractor, localization, harmonic balance, harmonic linearization, describing function method justification, absolute stability, counterexamples to Aizerman problem, conjecture, Kalman problem, hidden chaotic attractor, Chua circuit.

\section{Introduction}

The problem of hidden oscillations in nonlinear control systems forces to develop new approaches of nonlinear oscillation theory. During initial establishment and development of theory of nonlinear oscillations in the first half of 20th century (see [|Timoshenko(1928), van der Pol(1920), van der Pol(1926), Andronov et al.(1966), Stoker(1950)[]) a main attention has been given to analysis and synthesis of oscillating systems for which the solution of existence problems of oscillating regimes was not too difficult. The structure itself of many systems was such that they had oscillating solutions, the existence of which was "almost obvious". The arising in these systems periodic solutions were well seen by numerical analysis when numerical integration procedure of the trajectories allowed one to pass from small neighborhood of equilibrium to periodic trajectory. Therefore main attention of researchers was concentrated on analysis of forms and properties of these oscillations (the "almost" harmonic, relaxation, synchronous, circular, orbitally stable ones, and so on).

Further there came to light so called hidden oscillations - the oscillations, the existence itself of which is not obvious (which are "small" and, therefore, are difficult for numerical analysis or are not "connected" with equilibrium i.e. the creation of numerical procedure of integration of trajectories for the passage from equilibrium to periodic solution is impossible). The simplest examples of such hidden oscillations are family of nested small or large limit cycles in two-dimension dynamical systems (see, e.g., [|Kuznetsov \& Leonov(2008), Leonov(2010), Leonov et al.(2011)|]]).

In the midpoint of twentieth century M.A.Aizerman [|Aizerman(1949)|] and R.E. Kalman [|Kalman(1957)|] formulated two conjectures, which occupy, at once, attention of many famous scholars [[Krasovsky(1952), Malkin(1952), Erugin(1952), Pliss(1958), Lefschetz(1965), Barabanov et al.(1996)|]. The attempts to refute these conjectures lead to creation of effective methods for the search of hidden oscillations.

\footnotetext{
${ }^{1}$ Nikolay V. Kuznetsov, nkuznetsov239 at gmail.com (correspondence author)

${ }^{2} \mathrm{PDF}$ slides http://www.math.spbu.ru/user/nk/PDF/Hidden-oscillation-Absolute-stability-Aizerman-problem-Kalman.pdf

${ }^{3} \mathrm{PDF}$ slides http://www.math.spbu.ru/user/nk/PDF/Hidden-attractor-localization-Chua-circuit.pdf
} 
In this work these effective methods are described and a new approach for the study of hidden oscillations, based on the union of analytical and numerical methods, is considered. These methods allow to localize not only hidden oscillations, but also hidden attractors [|Leonov(2006), Leonov et al.(1995), Leonov \& Kuznetsov(2007), Leonov(2008a), Leonov(2008b)|]. In this work localization of hidden attractors (a basin of attraction of which does not contain neighborhoods of equilibria) in Chua's systems is demonstrated.

\section{Analytical-numerical method for hidden oscillation localization}

In the works [|Leonov(2009a), Leonov(2009b), Leonov(2009c), Leonov(2010)|] the methods of search of periodic solutions of multidimensional nonlinear dynamical systems with scalar nonlinearity were suggested. In the present work the approach suggested is generalized on the systems of the form

$$
\frac{d \mathbf{x}}{d t}=\mathbf{P} \mathbf{x}+\boldsymbol{\psi}(\mathbf{x})
$$

where $\mathbf{P}$ is a constant $n \times n$-matrix, $\boldsymbol{\psi}(\mathbf{x})$ is a continuous vector-function, and $\boldsymbol{\psi}(0)=0$.

For the search of periodic solution close to harmonic oscillation, we consider matrix $\mathbf{K}$ such that the matrix $\mathbf{P}_{0}=\mathbf{P}+\mathbf{K}$ has a pair of purely imaginary eigenvalues $\pm i \omega_{0}\left(\omega_{0}>0\right)$ and the rest of its eigenvalues have negative real parts. Then system (2.1) can be rewritten as

$$
\frac{d \mathbf{x}}{d t}=\mathbf{P}_{0} \mathbf{x}+\varphi(\mathbf{x})
$$

where $\boldsymbol{\varphi}(\mathrm{x})=\boldsymbol{\psi}(\mathrm{x})-\mathbf{K} \mathbf{x}$.

Since we are interested in periodic solutions of system (2.2), it is natural to introduce a finite sequence of continuous functions $\varphi^{0}(\mathbf{x}), \varphi^{1}(\mathbf{x}), \ldots, \varphi^{m}(\mathbf{x})$ in such a way that the graphs of neighboring functions $\varphi^{j}$ and $\boldsymbol{\varphi}^{j+1}$ in a sense, are slightly differ from each other, the function $\varphi^{0}(\mathbf{x})$ is small, and $\varphi^{m}(\mathbf{x})=\boldsymbol{\varphi}(\mathbf{x})$.

In this case the smallness of function $\varphi^{0}(\mathbf{x})$ permits one to apply and justify the method of harmonic linearization for the system

$$
\frac{d \mathbf{x}}{d t}=\mathbf{P}_{0} \mathbf{x}+\varphi^{0}(\mathbf{x})
$$

if the stable periodic solution $\mathbf{x}^{0}(t)$ close to harmonic one is determined. All the points of this stable periodic solution are located in the domain of attraction of stable periodic solution $\mathbf{x}^{1}(t)$ of the system

$$
\frac{d \mathbf{x}}{d t}=\mathbf{P}_{0} \mathbf{x}+\varphi^{j}(\mathbf{x})
$$

with $j=1$ or when passing from (2.3) to system (2.4) with $j=1$, we observe the instability bifurcation destroying periodic solution. In the first case it is possible to find $\mathbf{x}^{1}(t)$ numerically, starting a trajectory of system (2.4) with $j=1$ from the initial point $\mathbf{x}^{0}(0)$.

Starting from the point $\mathbf{x}^{0}(0)$, after transient process the computational procedure reaches the periodic solution $\mathbf{x}^{1}(t)$ and computes it. In this case the interval $(0, \widetilde{T}]$, on which the computation is carried out, must be sufficiently large.

After the computation of $\mathbf{x}^{1}(t)$ it is possible to obtain the following system (2.4) with $j=2$ and to organize a similar procedure of computing the periodic solution $\mathbf{x}^{2}(t)$, starting a trajectory, which with increasing $t$ approaches to periodic trajectory $\mathbf{x}^{2}(t)$, from the initial point $\mathbf{x}^{2}(0)=\mathbf{x}^{1}(\widetilde{T})$.

Proceeding this procedure and computing $\mathbf{x}^{j}(t)$, using trajectories of system (2.4) with the initial data $\mathbf{x}^{j}(0)=\mathbf{x}^{j-1}(\widetilde{T})$, we either arrive at periodic solution of system (2.4) with $j=m$ (i.e., at original system $(2.2))$ or observe, at a certain step, the instability bifurcation destroying periodic solution. 
For system (2.3) with such function $\varphi^{0}(\mathbf{x})$ it turns out that it is possible to justify rigorously the method of harmonic linearization and to determine the initial conditions, for which system (2.3) has a stable periodic solution close to harmonic one.

Note that at some step the procedure may reach a locally stable attractor. This effect will be demonstrated here for Chua's systems.

\subsection{System reduction}

By nonsingular linear transformation $\mathbf{x}=$ Sy system (2.3) can be reduced to the form

$$
\begin{aligned}
& \dot{y}_{1}=-\omega_{0} y_{2}+\phi_{1}\left(y_{1}, y_{2}, \mathbf{y}_{3}\right) \\
& \dot{y}_{2}=\omega_{0} y_{1}+\phi_{2}\left(y_{1}, y_{2}, \mathbf{y}_{3}\right) \\
& \dot{\mathbf{y}}_{3}=\mathbf{A y}_{3}+\phi_{3}\left(y_{1}, y_{2}, \mathbf{y}_{3}\right)
\end{aligned}
$$

where $\phi_{3}$ is an $(n-2)$-dimensional vector-function, $\phi_{1}, \phi_{2}$ are certain scalar functions; $y_{1}$ and $y_{2}$ are scalars, $\mathbf{y}_{3}$ is an $(n-2)$-dimensional vector.

Here $\mathbf{A}$ is a constant $(n-2) \times(n-2)$ matrix, all eigenvalues of which have negative real parts. Without loss of generality, it may be assumed that for the matrix $\mathbf{A}$ there exists positive number $d>0$ such that

$$
\mathbf{y}_{3}^{*}\left(\mathbf{A}+\mathbf{A}^{*}\right) \mathbf{y}_{3} \leq-2 d\left|\mathbf{y}_{3}\right|^{2}, \quad \forall \mathbf{y}_{3} \in \mathbb{R}^{n-2}
$$

Here $*$ is a transposition operation.

For scalar case in (2.1) we have $\boldsymbol{\psi}(\mathbf{y})=\mathbf{q} \varphi\left(\mathbf{r}^{*} \mathbf{y}\right)$ and $\mathbf{K}=k \mathbf{q} \mathbf{r}^{*}$, where $\mathbf{r}$ and $\mathbf{q}$ are $n$-dimensional vectors, $\varphi(\sigma)$ is a continuous scalar function $(\varphi(0)=0), k$ is a coefficient of harmonic linearization. Here it is always possible by nonsingular linear transformation to reduce the system in a such way that

$$
\begin{aligned}
& \phi_{1}\left(y_{1}, y_{2}, \mathbf{y}_{3}\right)=b_{1} \varphi\left(y_{1}+\mathbf{c}^{*} \mathbf{y}_{3}\right) \\
& \phi_{2}\left(y_{1}, y_{2}, \mathbf{y}_{3}\right)=b_{2} \varphi\left(y_{1}+\mathbf{c}^{*} \mathbf{y}_{3}\right) \\
& \boldsymbol{\phi}_{3}\left(y_{1}, y_{2}, \mathbf{y}_{3}\right)=\mathbf{b} \varphi\left(y_{1}+\mathbf{c}^{*} \mathbf{y}_{3}\right)
\end{aligned}
$$

where $\mathbf{b}$ and $\mathbf{c}$ are $(n-2)$-dimensional vectors, $b_{1}$ and $b_{2}$ are certain real numbers.

In scalar case one can write out the transfer function of system (2.2):

$$
W_{1}(p)=\mathbf{r}^{*}\left(\mathbf{P}_{0}-p \mathbf{I}\right)^{-1} \mathbf{q}=\frac{\eta p+\theta}{p^{2}+\omega_{0}^{2}}+\frac{R(p)}{Q(p)},
$$

and the transfer function of system (2.5):

$$
W_{2}(p)=\frac{-b_{1} p+b_{2} \omega_{0}}{p^{2}+\omega_{0}^{2}}+\mathbf{c}^{*}(\mathbf{A}-p \mathbf{I})^{-1} \mathbf{b} .
$$

Here $\eta, \theta$ are certain real numbers, $Q(p)$ is a stable polynomial of degree $(n-2), R(p)$ is a polynomial of degree smaller than $(n-2)$. Suppose, the polynomials $R(p)$ and $Q(p)$ have no common roots. By equivalence of systems $(2.2)$ and (2.5) the transfer functions of these systems coincide. This implies the relations

$$
\begin{aligned}
& \eta=-b_{1}, \theta=b_{2} \omega_{0}, \mathbf{c}^{*} \mathbf{b}+b_{1}=\mathbf{r}^{*} \mathbf{q}=-\lim _{p \rightarrow \infty} p W_{1}(p), \\
& \frac{R(p)}{Q(p)}=\mathbf{c}^{*}(\mathbf{A}-p \mathbf{I})^{-1} \mathbf{b} .
\end{aligned}
$$




\section{Poincare map for Harmonic linearization in noncritical case}

Consider system (2.5) with nonlinearity $\phi=\varepsilon \varphi$, where $\varepsilon$ is "classical" small positive parameter.

Suppose, for the vector-function $\varphi(\mathbf{y})$ the estimate

$$
\left|\varphi\left(\mathbf{y}^{\prime}\right)-\varphi\left(\mathbf{y}^{\prime \prime}\right)\right| \leq L\left|\mathbf{y}^{\prime}-\mathbf{y}^{\prime \prime}\right|, \quad \forall \mathbf{y}^{\prime}, \mathbf{y}^{\prime \prime} \in \mathbb{R}^{n}
$$

is satisfied.

In a phase space of system (2.5) we introduce the following set

$$
\Omega=\left\{\left|\mathbf{y}_{3}\right| \leq D \varepsilon, \quad y_{2}=0, \quad y_{1} \in\left[a_{1}, a_{2}\right]\right\} .
$$

Here $D, a_{1}, a_{2}$ are certain positive numbers, which will be determined below.

Define $n$-dimensional vector $\mathbf{O}_{\mathbf{n}}(\varepsilon)$ as

$$
\mathbf{O}_{\mathbf{n}}(\varepsilon)=\left(\begin{array}{c}
O(\varepsilon) \\
\vdots \\
O(\varepsilon)
\end{array}\right)
$$

From condition (3.11) and the form of system (2.5) for solutions with initial data from $\Omega$ we obtain the following relations

$$
\begin{aligned}
& y_{1}(t)=\cos \left(\omega_{0} t\right) y_{1}(0)+O(\varepsilon) \\
& y_{2}(t)=\sin \left(\omega_{0} t\right) y_{1}(0)+O(\varepsilon), \\
& \mathbf{y}_{3}(t)=\exp (\mathbf{A} t) \mathbf{y}_{3}(0)+\mathbf{O}_{\mathbf{n}-\mathbf{2}}(\varepsilon) .
\end{aligned}
$$

From formulas (3.13) it follows that for any point $\left(y_{1}(0), y_{2}(0)=0, \mathbf{y}_{3}(0)\right)$, belonging to $\Omega$, there exists a number

$$
T=T\left(y_{1}(0), \mathbf{y}_{3}(0)\right)=2 \pi / \omega_{0}+O(\varepsilon)
$$

such that relations

$$
y_{1}(T)>0, \quad y_{2}(T)=0
$$

are satisfied and conditions

$$
y_{1}(t)>0, y_{2}(t)=0, \quad \forall t \in(0, T)
$$

are not satisfied.

Construct a Poincare map $F$ of the set $\Omega$ for the trajectories of system (2.5):

$$
F\left\|\begin{array}{c}
y_{1}(0) \\
0 \\
\mathbf{y}_{3}(0)
\end{array}\right\|=\left\|\begin{array}{c}
y_{1}(T) \\
0 \\
\mathbf{y}_{3}(T)
\end{array}\right\| .
$$

Introduce the describing function

$$
\begin{aligned}
& \Phi(a)=\int_{0}^{2 \pi / \omega_{0}}\left[\varphi_{1}\left(\left(\cos \omega_{0} t\right) a,\left(\sin \omega_{0} t\right) a, 0\right) \cos \omega_{0} t+\right. \\
& \left.+\varphi_{2}\left(\left(\cos \omega_{0} t\right) a,\left(\sin \omega_{0} t\right) a, 0\right) \sin \omega_{0} t\right] d t .
\end{aligned}
$$

From estimates (3.13) and condition on nonlinearity (3.11) for solutions of system (2.5) we obtain the following relations

$$
\begin{aligned}
& \left|\mathbf{y}_{3}(T)\right| \leq D \varepsilon, \\
& y_{1}^{2}(T)-y_{1}^{2}(0)=2 y_{1}(0) \varepsilon \Phi\left(y_{1}(0)\right)+O\left(\varepsilon^{2}\right) .
\end{aligned}
$$


Theorem 1 If the inequalities

$$
\Phi\left(a_{1}\right)>0, \quad \Phi\left(a_{2}\right)<0
$$

are satisfied, then for small enough $\varepsilon>0$ the Poincare map $F$ of the set $\Omega$ into itself is as follows

$$
F \Omega \subset \Omega .
$$

From this theorem and the Brouwer fixed point theorem we have the following

Theorem 2 If the inequalities (3.17) are satisfied, then for small enough $\varepsilon>0$ system (2.5) has a periodic solution with the period

$$
T=\frac{2 \pi}{\omega_{0}}+O(\varepsilon) .
$$

This solution is stable in the sense that its neighborhood $\Omega$ is mapped into itself: $F \Omega \subset \Omega$.

Corollary 1 If the conditions

$$
\Phi\left(a_{0}\right)=0,\left.\eta \frac{d \Phi(a)}{d a}\right|_{a=a_{0}}>0
$$

are satisfied, then for small enough $\varepsilon>0$ system with scalar nonlinearity and with transfer function (2.8) has T-periodic solution such that

$$
\mathbf{r}^{*} \mathbf{y}(t)=a_{0} \cos \left(\omega_{0} t\right)+O(\varepsilon), \quad T=\frac{2 \pi}{\omega_{0}}+O(\varepsilon) .
$$

Corollary 1 formally coincide with the procedure of search of stable periodic solutions by means of the harmonic linearization method [|Khalil(2002)|] (in noncritical case - nonlinearity does not belong to stability sector).

\section{Hidden attractor localization in Chua's system}

Let us apply the above algorithm to fulfil localization of attractors of the systems, which were obtained by Chua and his progenies in studying nonlinear electrical circuits with feedback [|Chua \& Lin(1990), Chua(1992a), Chua(1992b), Bilotta \& Pantano(2008), Chen \& Ueta(2002)|]. The systems of differential equations, describing the behavior of Chua's circuits, are three-dimensional dynamical systems with scalar nonlinearity.

Let us consider Chua's system represented in dimensionless quantities.

$$
\begin{aligned}
& \dot{x}=\alpha(y-x)-\alpha \psi(x), \\
& \dot{y}=x-y+z, \\
& \dot{z}=-(\beta y+\gamma z) .
\end{aligned}
$$

Here the function

$$
\begin{aligned}
& \psi(x)=m_{1} x+\left(m_{0}-m_{1}\right) \operatorname{sat}(x)= \\
& m_{1} x+\frac{1}{2}\left(m_{0}-m_{1}\right)(|x+1|-|x-1|)
\end{aligned}
$$

describes a nonlinear element of system, it is also called Chua's diode, $\alpha, \beta, \gamma, m_{0}, m_{1}$ are parameters of the classical Chua's system.

For attractor localization of this system we plug-in [|Kuznetsov et al.(2010)|] the coefficient $k$ and the small parameter $\varepsilon$ into system (4.18) and construct solutions of system (4.18) with the nonlinearity $\varepsilon \varphi(x)=$ $\varepsilon(\psi(x)-k x)$ by means of sequential increasing $\varepsilon$ with the step 0.1 from the value $\varepsilon_{1}=0.1$ to $\varepsilon_{10}=1$. 
Corollary 1 allows to compute the initial data for the system (2.5). We have to calculate the matrix $\mathbf{S}$ transforming system (2.1) into (2.5) to obtain the initial data for the system (2.1).

Rewrite Chua's system as a Lur'e system

$$
\frac{d \mathbf{x}}{d t}=\mathbf{P} \mathbf{x}+\mathbf{q} \psi\left(\mathbf{r}^{*} \mathbf{x}\right)
$$

where

$$
\begin{gathered}
\mathbf{x} \in \mathbb{R}^{3}, \mathbf{P}=\left(\begin{array}{ccc}
-\alpha\left(m_{1}+1\right) & \alpha & 0 \\
1 & -1 & 1 \\
0 & -\beta & -\gamma
\end{array}\right), \mathbf{q}=\left(\begin{array}{c}
-\alpha \\
0 \\
0
\end{array}\right) \\
\mathbf{r}=\left(\begin{array}{l}
1 \\
0 \\
0
\end{array}\right) .
\end{gathered}
$$

Further consider the coefficient $k$, small parameter $\varepsilon$ and rewrite system (4.20) in the form of (2.3)

$$
\frac{d \mathbf{x}}{d t}=\mathbf{P}_{0} \mathbf{x}+\mathbf{q} \varepsilon \varphi\left(\mathbf{r}^{*} \mathbf{x}\right)
$$

where

$$
\begin{array}{r}
\mathbf{P}_{\mathbf{0}}=\mathbf{P}+k \mathbf{q r}^{*}=\left(\begin{array}{ccc}
-\alpha\left(m_{1}+1+k\right) & \alpha & 0 \\
1 & -1 & 1 \\
0 & -\beta & -\gamma
\end{array}\right), \\
\lambda_{1,2}^{\mathbf{P}_{0}}= \pm i \omega_{0}, \\
\quad \lambda_{3}^{\mathbf{P}_{0}}=-d<0, \\
\varphi(\sigma)=\psi(\sigma)-k \sigma .
\end{array}
$$

Using a nonsingular linear transformation $\mathbf{x}=\mathbf{S y}$, it is possible to transform system (4.21) into the form

$$
\frac{d \mathbf{y}}{d t}=\mathbf{H y}+\mathbf{b} \varepsilon \varphi\left(\mathbf{u}^{*} \mathbf{y}\right)
$$

where

$$
\mathbf{H}=\left(\begin{array}{ccc}
0 & -\omega_{0} & 0 \\
\omega_{0} & 0 & 0 \\
0 & 0 & -d
\end{array}\right), \mathbf{b}=\left(\begin{array}{c}
b_{1} \\
b_{2} \\
1
\end{array}\right), \mathbf{u}=\left(\begin{array}{c}
1 \\
0 \\
-h
\end{array}\right)
$$

Here the transfer functions $W_{\mathbf{H}}(p)$ of system (4.22) can be represented as

$$
W_{\mathbf{H}}(p)=\frac{-b_{1} p+b_{2} \omega_{0}}{p^{2}+\omega_{0}^{2}}+\frac{h}{p+d} .
$$

Then, using the equality of transfer functions

$$
W_{\mathbf{H}}(p)=\mathbf{r}^{*}\left(\mathbf{P}_{0}-p \mathbf{I}\right)^{-1} \mathbf{q}
$$

of system (4.21) and system (4.22), one can obtain

$$
\begin{aligned}
& k=\frac{-\alpha\left(m_{1}+m_{1} \gamma+\gamma\right)+\omega_{0}^{2}-\gamma-\beta}{\alpha(1+\gamma)}, \\
& d=\frac{\alpha+\omega_{0}^{2}-\beta+1+\gamma+\gamma^{2}}{1+\gamma} \\
& h=\frac{\alpha\left(\gamma+\beta-(1+\gamma) d+d^{2}\right)}{\omega_{0}^{2}+d^{2}} \\
& b_{1}=\frac{\alpha\left(\gamma+\beta-\omega_{0}^{2}-(1+\gamma) d\right)}{\omega_{0}^{2}+d^{2}} \\
& b_{2}=\frac{\alpha\left((1+\gamma-d) \omega_{0}^{2}+(\gamma+\beta) d\right)}{\omega_{0}\left(\omega_{0}^{2}+d^{2}\right)} .
\end{aligned}
$$


Since system (4.21) transforms into system (4.22) by nonsingular linear conversion $\mathbf{x}=\mathbf{S y}$, therefore, the matrix $\mathbf{S}$ satisfies the following equations

$$
\mathbf{H}=\mathbf{S}^{-1} \mathbf{P}_{\mathbf{0}} \mathbf{S}, \quad \mathbf{b}=\mathbf{S}^{-1} \mathbf{q}, \quad \mathbf{u}^{*}=\mathbf{r}^{*} \mathbf{S} .
$$

Solving these matrix equations, for the matrix

$$
\mathbf{S}=\left(\begin{array}{lll}
s_{11} & s_{12} & s_{13} \\
s_{21} & s_{22} & s_{23} \\
s_{31} & s_{32} & s_{33}
\end{array}\right)
$$

we obtain

$$
\begin{aligned}
& s_{11}=1, \quad s_{12}=0, \quad s_{13}=-h \\
& s_{21}=m_{1}+1+k, s_{22}=-\frac{\omega_{0}}{\alpha}, \\
& s_{23}=-\frac{h\left(\alpha\left(m_{1}+1+k\right)-d\right)}{\alpha} \\
& s_{31}=\frac{\alpha\left(m_{1}+k\right)-\omega_{0}^{2}}{\alpha}, \\
& s_{32}=-\frac{\alpha(\beta+\gamma)\left(m_{1}+k\right)+\alpha \beta-\gamma \omega_{0}^{2}}{\alpha \omega_{0}} \\
& s_{33}=h \frac{\alpha\left(m_{1}+k\right)(d-1)+d(1+\alpha-d)}{\alpha} .
\end{aligned}
$$

For small enough $\varepsilon$ one can obtain the initial data

$$
\mathbf{y}(0)=\left(\begin{array}{c}
y_{1}(0) \\
y_{2}(0) \\
\mathbf{y}_{3}(0)
\end{array}\right)=\left(\begin{array}{c}
a_{0} \\
0 \\
0
\end{array}\right)
$$

for the first step of the multistep procedure for localization of hidden oscillation. By (4.25) we obtain the relations between the initial data of systems (4.21) and (4.22)

$$
\mathbf{x}(0)=\mathbf{S y}(0)=\mathbf{S}\left(\begin{array}{c}
a_{0} \\
0 \\
0
\end{array}\right)=\left(\begin{array}{c}
a_{0} s_{11} \\
a_{0} s_{21} \\
a_{0} s_{31}
\end{array}\right)
$$

Returning to the notions of Chua's system, we obtain the following formulae for defining initial data:

$$
x(0)=a_{0}, y(0)=a_{0}\left(m_{1}+1+k\right), z(0)=a_{0} \frac{\alpha\left(m_{1}+k\right)-\omega_{0}^{2}}{\alpha} .
$$

Thus, using the obtained relations for initial data and applying the multistage algorithm described above, we can numerically simulate Chua's system, represented in the form (2.5).

Let us apply localization procedure, described above. Here the simplest and the most natural class of functions $\varphi^{j}$ are the following functions: $\varphi^{1}(\sigma)=\varepsilon_{1} \varphi(\sigma), \ldots, \varphi m-1(\sigma)=\varepsilon_{m-1} \varphi(\sigma), \varphi^{m}(\sigma)=\varphi(\sigma)$, where $\varepsilon_{j}=j / m, j=1, \ldots, m$.

Consider an example $\alpha=8.4562, \beta=12.0732, \gamma=0.0052, m_{0}=-0.1768, m_{1}=-1.1468$.

Firstly, we compute the coefficient of harmonic linearization $k=0.2098$ and the value of "start" frequency $\omega_{0}=2.0392$. Using relations (4.28) we obtain initial data $x(0)=-49.9061, y(0)=-3.1469, z(0)=71.2994$ for the first step of multistage procedure of construction of solutions. For $\varepsilon_{1}=0.1$ after transient process the computational procedure arrives at a periodic solution close to harmonic one. Further with increasing parameter $\varepsilon$ this periodic solution close to harmonic one is transformed into chaotic attractor.

In the typical Chua's system there occurs classical excitation of oscillations in the case when a trajectory from the neighborhood of unstable zero equilibrium reaches the attractor. In this system, in despite of the existence of stable zero equilibrium, the described procedure also allows one to go on "hidden" attractor by means of sequential approximations.

The projections of solution on the plane $\{x, y\}$ and transformation of the nonlinearity from the unstable sector up to the stable one for the values $\varepsilon_{1}=0.1$ and $\varepsilon_{10}=1$ respectively are shown in the Fig. 2 . 

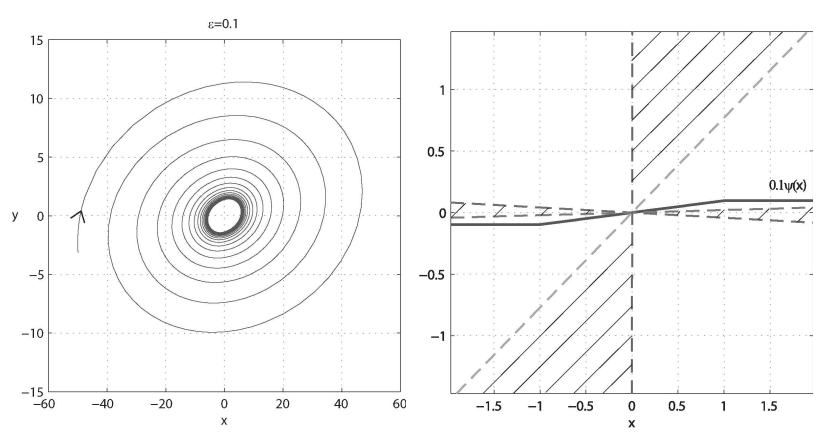

Figure 1: System with $\varepsilon=0.1$ : trajectory projection on $(x, y)$; stability sector (shaded) and nonlinearity $\varphi^{1}(\sigma)$.
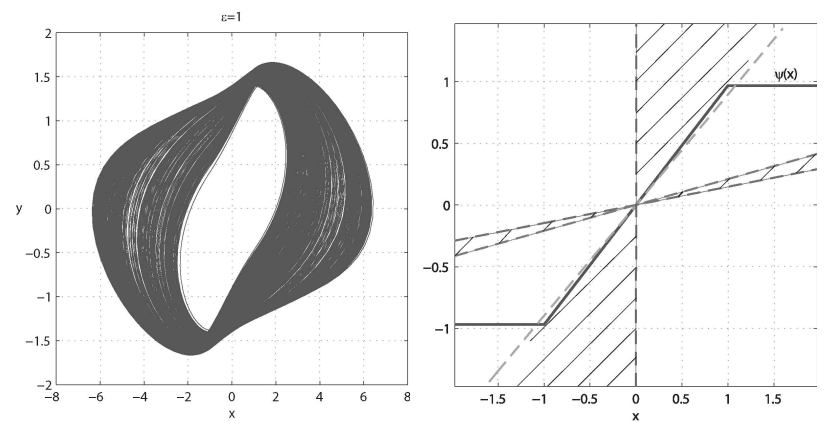

Figure 2: System with $\varepsilon=1$ : trajectory projection on $(x, y)$; stability sector and nonlinearity $\varphi^{10}(\sigma)=\operatorname{sat}(\sigma)$.

\subsection{Generalized Chua's system with scalar nonlinearity}

Let us consider system (4.18) with following nonlinearity (generalized Chua's system)

$$
\begin{aligned}
\psi(x) & =m_{1} x+\left(m_{0}-m_{1}\right) \operatorname{sat}(x)+ \\
& \left.+\frac{1}{2}\left(s-m_{0}\right)\left(\left|x+\delta_{0}\right|-\left|x-\delta_{0}\right|\right)\right),
\end{aligned}
$$

where $\alpha, \beta, \gamma, m_{0}, m_{1}$ are parameters of the classical Chua's system, and $\delta_{0}$ and $s$ are parameters that determine the stability of zero equilibrium.

Modified and classical nonlinearities are shown in the Fig. 3. The shaded area is the stability sector.

A similar idea of nonlinearity modification was applied in the works [|Suykens et al.(1997), Savaci \& Gunel(2006) to increase the number of scrolls of attractor without increase of the system's order.

The procedure described above can be used for numerical localization of attractor of the generalized Chua's system.

Here

$$
\begin{aligned}
& \mathbf{P}_{\mathbf{0}}=\mathbf{P}+k \mathbf{q r}^{*}=\left(\begin{array}{ccc}
-\alpha(1+k) & \alpha & 0 \\
1 & -1 & 1 \\
0 & -\beta & -\gamma
\end{array}\right), \\
& k=\frac{-\alpha \gamma+\omega_{0}^{2}-\gamma-\beta}{\alpha(1+\gamma)}
\end{aligned}
$$

and $d, h, b_{1}, b_{2}$ are as in (4.23).

Solving matrix equations (4.24), for the matrix $\mathbf{S}$ we obtain

$$
\begin{aligned}
& s_{11}=1, \quad s_{12}=0, \quad s_{13}=-h, \\
& s_{21}=1+k, \quad s_{22}=-\frac{\omega_{0}}{\alpha}, \quad s_{23}=\frac{h(d-k \alpha-\alpha)}{\alpha},
\end{aligned}
$$




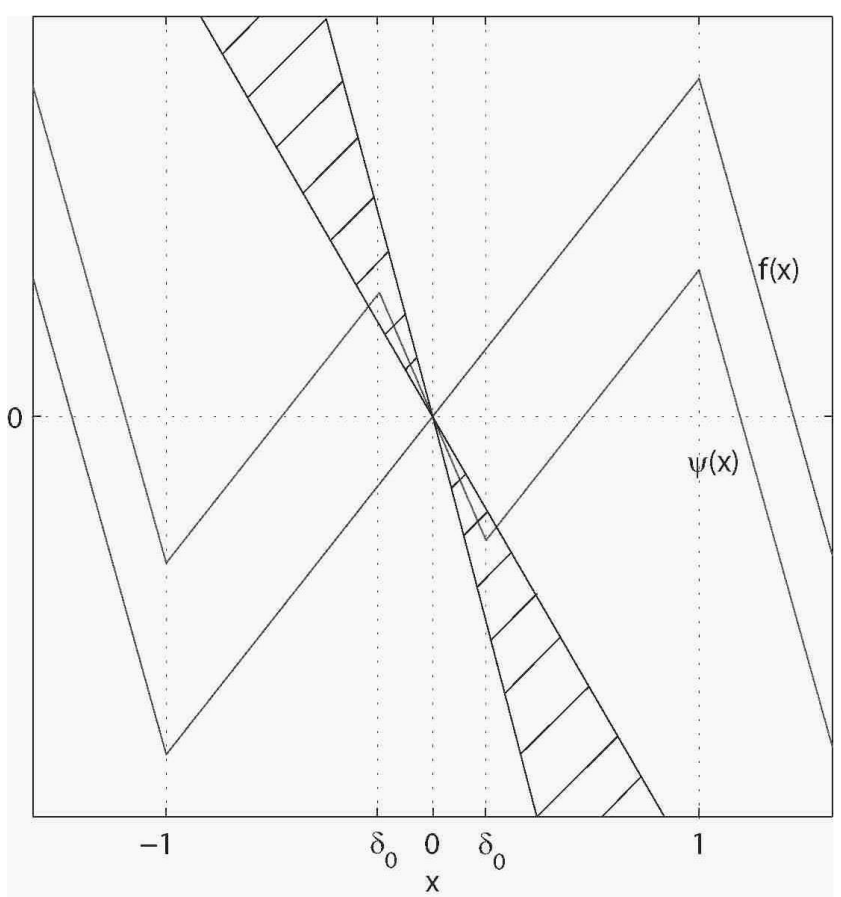

Figure 3: Standard Chua's diode $f(x)$ and modified nonlinearity $\psi(x)$ with stability sector.

$$
\begin{gathered}
s_{31}=\frac{k \alpha-\omega_{0}^{2}}{\alpha}, \quad s_{32}=-\frac{\left(\alpha \beta+k \alpha \beta+k \alpha \gamma-\gamma \omega_{0}^{2}\right)}{\alpha \omega_{0}}, \\
s_{33}=-\frac{h\left(d^{2}-(1+\alpha+k \alpha) d+k \alpha\right)}{\alpha} .
\end{gathered}
$$

For small enough $\varepsilon$ one can obtain the initial data

$$
x(0)=a_{0}, \quad y(0)=a_{0}(1+k), \quad z(0)=a_{0} \frac{k \alpha-\omega_{0}^{2}}{\alpha} .
$$

for the first step of the multistep procedure for localization of hidden oscillation.

Let us apply localization procedure, described above. Consider an example $s=-0.31, \delta_{0}=0.2, a=$ $0.1691, b=-0.4768, \alpha=-1.398, \beta=-0.0136, \gamma=-0.0297$.

Firstly, we compute the coefficient of harmonic linearization $k=-0.3067$ and the value of "start" frequency $\omega_{0}=0.6436$. Using relations $(4.28)$ we obtain initial data $x(0)=-1.1061, y(0)=-0.7669, z(0)=0.0115$ for the first step of multistage procedure of construction of solutions. For $\varepsilon_{1}=0.1$ after transient process the computational procedure arrives at an almost periodic solution close to harmonic one. Further, with increasing parameter $\varepsilon$ this periodic solution will be transformed into chaotic attractor of the type "doublescroll" [|Bilotta \& Pantano(2008)|].

In this system, in despite of the existence of stable zero equilibrium, the described procedure also allows one to go on "hidden" attractor by means of sequential approximations. The projections of solutions on the plane $\{x, y\}$ for the values $\varepsilon_{1}=0.1, \varepsilon_{3}=0.3, \varepsilon_{7}=0.7$, and $\varepsilon_{10}=1$, are shown in the Fig. 4 respectively.

\subsection{Generalized Chua's system with vector nonlinearity}

Consider modification of Chua's system

$$
\begin{aligned}
& \dot{x}=\alpha(y-x)-\alpha f_{m}(x), \\
& \dot{y}=x-y+z+g(y), \\
& \dot{z}=-(\beta y+\gamma z),
\end{aligned}
$$



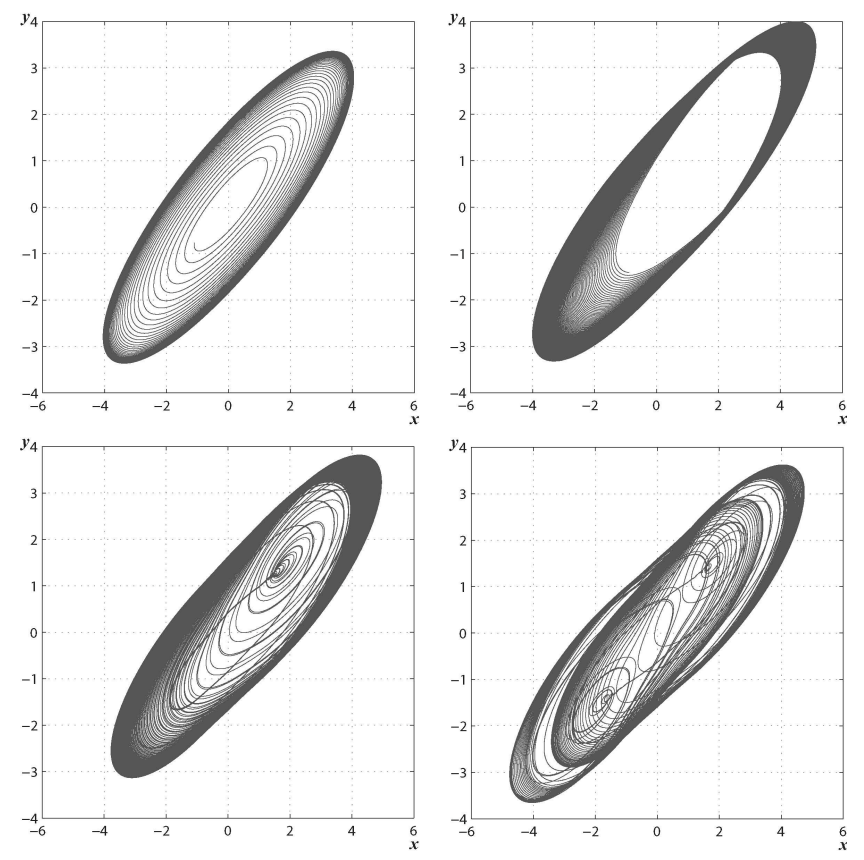

Figure 4: $\varepsilon=0.1, \varepsilon=0.3, \varepsilon=0.7, \varepsilon=1$

where

$$
f_{m}(x)=\left(k_{1} x+k_{3} x^{3}+k_{5} x^{5}\right), \quad g(x)=c y^{2} .
$$

Let $k_{1}=-0.3092, k_{3}=0.6316, k_{5}=-0.3$, then zero solution of system (4.29) is stable.

Taking $\omega_{0}=2.5, d=10$ (so we define matrix $\mathbf{H}$, and one can obtain linearization matrix $\mathbf{K}$ ), the above procedure allows us to get initial data $x(0)=-1.5728, y(0)=0, z(0)=0$ for the first step of multistage procedure of construction of solutions. For $\varepsilon_{1}=0.1$ after transient process the computational procedure arrives at a almost periodic solution close to harmonic one. Further, with increasing parameter $\varepsilon$ this periodic solution will be transformed into hidden attractor.
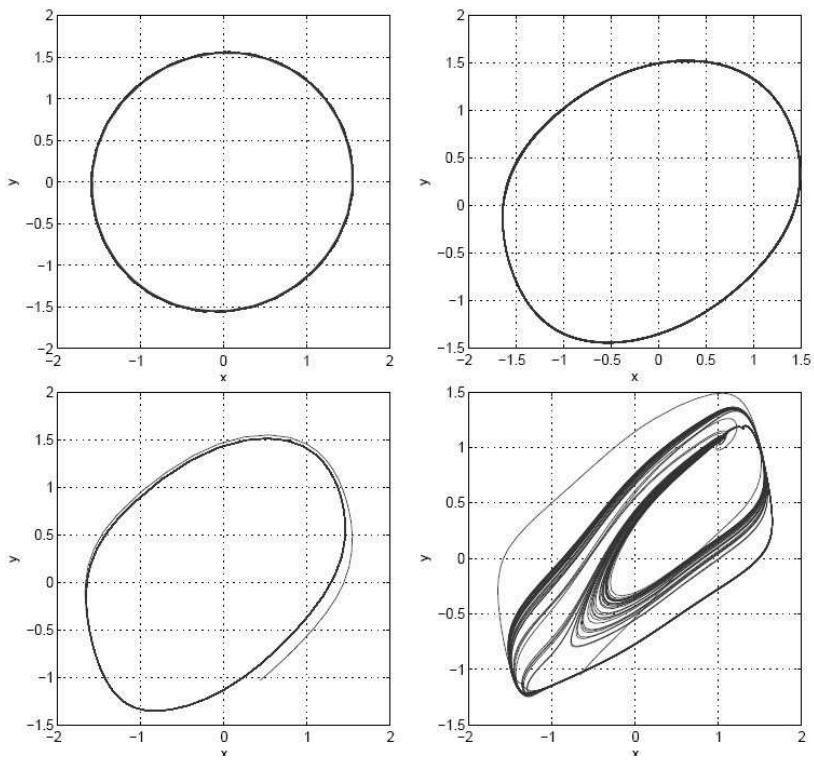

Figure 5: $\varepsilon=0.1, \varepsilon=0.5, \varepsilon=0.7, \varepsilon=1$ 


\section{Poincare map for Harmonic linearization in critical case. Aiz- erman and Kalman problems}

Consider (2.1) with scalar nonlinearity

$$
\frac{d \mathbf{x}}{d t}=\mathbf{P} x+\mathbf{q} \varphi\left(\mathbf{r}^{*} \mathbf{x}\right), \quad \mathbf{x} \in \mathbb{R}^{n}
$$

where $\varphi(\sigma)$ is a continuous piecewise-differentiable scalar function and $\varphi(0)=0$. Suppose that for all $k \in$ $\left(\mu_{1}, \mu_{2}\right)$ a zero solution of system (5.31) with $\varphi(\sigma)=k \sigma$ is asymptotically stable in the large (i.e., a zero solution is Lyapunov stable and any solution of system (5.31) tends to zero as $t \rightarrow \infty$. In other words, a zero solution is a global attractor of system (5.31) with $\varphi(\sigma)=k \sigma)$.

In 1949 M.A. Aizerman formulated [|Aizerman(1949)|] the following conjecture: any system (5.31) with a nonlinearity, satisfying the property

$$
\mu_{1} \sigma<\varphi(\sigma)<\mu_{2} \sigma \quad \sigma \neq 0
$$

is stable in the large.

The necessary criteria of absolute stability [|Leonov et al.(1996)|] contradict this hypothesis.

In 1957 R.E. Kalman has formulated a similar hypothesis [|Kalman(1957)|] with more restrictive condition: if at the points of differentiability of $\varphi(\sigma)$ the condition

$$
\mu_{1}<\varphi^{\prime}(\sigma)<\mu_{2}
$$

is satisfied, then system (5.31) is stable in the large.

It is well known that this hypothesis is valid for $n=2$ and $n=3$ (see, e.g., |Leonov et al.(1996)|).

The only widely cited in literature counterexample to this hypothesis is due to Fitts [|Fitts(1966)|]. It is obtained by numerical modeling of system (5.31) for $n=4$ with the transfer function

$$
W(p)=\frac{p^{2}}{\left[(p+\beta)^{2}+0.9^{2}\right]\left[(p+\beta)^{2}+1.1^{2}\right]}
$$

and the cubic nonlinearity $\varphi(\sigma)=k \sigma^{3}$.

Below we describe a computer modeling of the Fitts' system. Reconstructing the system from transfer function (5.34) for $\beta=0.01$ and $k=10$, we obtain

$$
\begin{aligned}
\dot{x}_{1}= & x_{2}, \\
\dot{x}_{2}= & x_{3} \\
\dot{x}_{3}= & x_{4} \\
\dot{x}_{4}= & -0.9803 x_{1}-0.0404 x_{2}-2.0206 x_{3}-0.0400 x_{4} \\
& +\varphi\left(-x_{3}\right)
\end{aligned}
$$

where $\varphi(\sigma)=10 \sigma^{3}$.

Modeling this system with initial data $x_{1}(0)=85.1189, x_{2}(0)=0.9222, x_{3}(0)=-2.0577, x_{4}(0)=-2.6850$, we obtain a "periodic" solution (Fig. 6).

Thus, Fitts' experiments have revealed a periodic solution of system (5.35) for some values of parameters $\beta$ and $k$. However, it was shown in $[\mid \operatorname{Barabanov}(1980)$, Barabanov(1988)|] that the results of experiments performed by Fitts for some of the parameter values $\beta \in(0.572,0.75)$ are incorrect.

In [|Barabanov(1988)|] a proof of the existence of a system of the form (5.31) with $n=4$ for which Kalmans conjecture is false was presented; this is an "existence theorem" and needs to be carefully checked. 

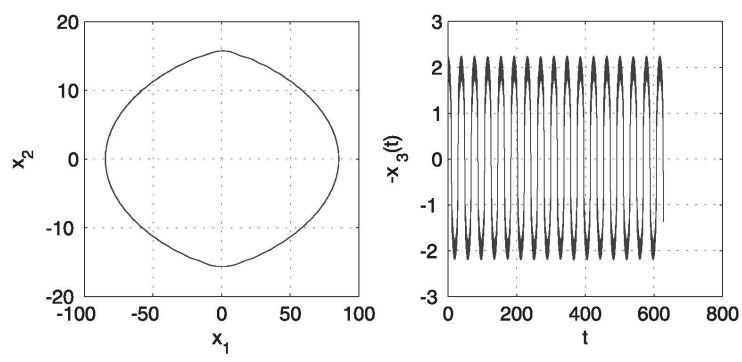

Figure 6: Trajectory of system (5.35) projection on $\left(x_{1}, x_{2}\right)$

In $|\operatorname{Barabanov}(1988)|$, the system

$$
\begin{aligned}
& \dot{x}_{1}=x_{2}, \\
& \dot{x}_{2}=-x_{4}, \\
& \dot{x}_{3}=x_{1}-2 x_{4}-\varphi\left(x_{4}\right), \quad \varphi(\sigma)=\operatorname{sign}(\sigma), \\
& \dot{x}_{4}=x_{1}+x_{3}-x_{4}-\varphi\left(x_{4}\right),
\end{aligned}
$$

was considered. To construct counterexample to Kalman's conjecture here one has to choose a nonlinearity "close" to $\operatorname{sign}(\sigma)$ and with positive derivative (because linear stability sector here is $(0, \mathrm{k})$ ). So we slightly change the form of this nonlinearity as

$$
\varphi(\sigma)= \begin{cases}5 \sigma, & \forall|\sigma| \leq \frac{1}{5} \\ \operatorname{sign}(\sigma)+\frac{1}{25}\left(\sigma-\operatorname{sign}(\sigma) \frac{1}{5}\right), & \forall|\sigma|>\frac{1}{5}\end{cases}
$$

The graph of this nonlinearity is shown in Fig. 7.

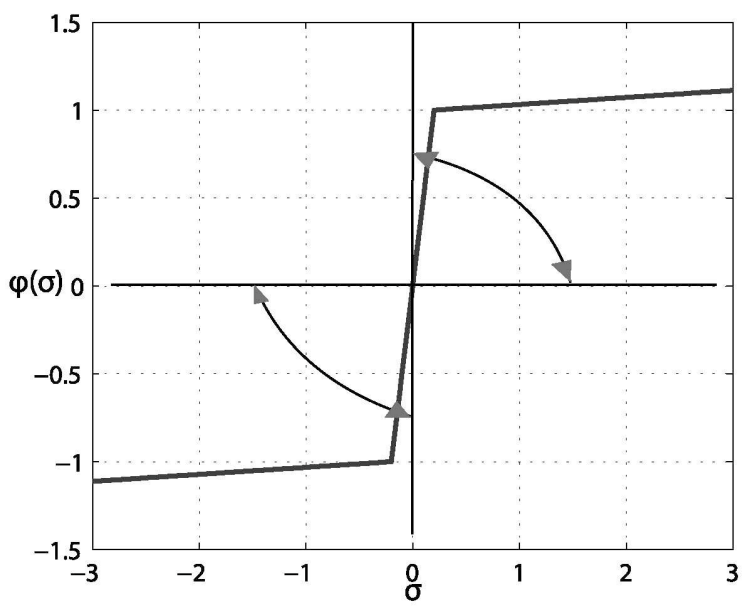

Figure 7: The graph of $\varphi(\sigma)$ and the stability sector

Let us find a periodic solution to system (5.36) with nonlinearity (5.37). Modeling this system with initial data $x_{1}(0)=0, x_{2}(0)=1 / 2, x_{3}(0)=0, x_{4}(0)=0$, we obtain the periodic solution shown in (Fig. 8).

In [|Bernat \& Llibre(1996), Meisters(1996), Glutsyuk(1997)|], "gaps" presented in [|Barabanov(1988)|] were pointed out: thus, in [|Bernat \& Llibre(1996)|], we read "He tried to prove that this system and systems close to this have a periodic orbit. But his arguments are not complete, and we checked numerically that in the region where he tries to find the periodic orbit all the solutions have $\omega$-limit equal to the origin". 

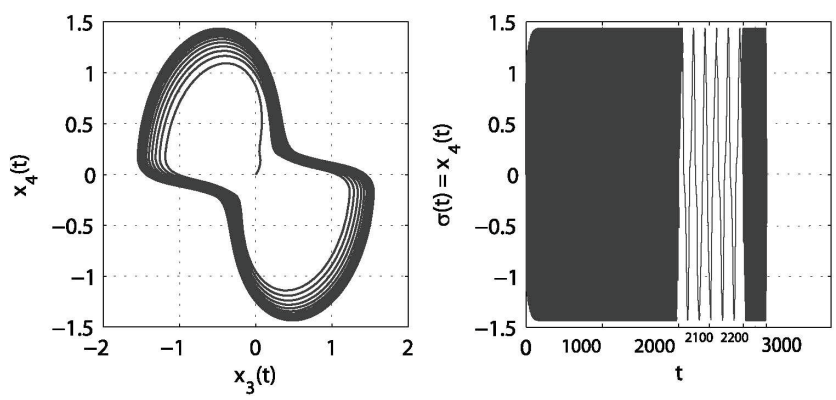

Figure 8: The projection of the trajectory of system (5.35) with initial data $x_{1}(0)=0, x_{2}(0)=1 / 2, x_{3}(0)=$ $0, x_{4}(0)=0$ on the plane $\left(x_{3}, x_{4}\right)$

In [|Bernat \& Llibre(1996)|] an attempt to overcome these "gaps" by analytical-numerical methods was made.

Let us model the system proposed in [[Bernat \& Llibre(1996)|], namely,

$$
\begin{aligned}
& \dot{x}_{1}=x_{2} \\
& \dot{x}_{2}=-x_{4} \\
& \dot{x}_{3}=x_{1}-2 x_{4}-\frac{9131}{900} \varphi\left(x_{4}\right) \\
& \dot{x}_{4}=x_{1}+x_{3}-x_{4}-\frac{1837}{180} \varphi\left(x_{4}\right),
\end{aligned}
$$

where

$$
\varphi(\sigma)= \begin{cases}\sigma, & \forall|\sigma| \leq \frac{900}{9185} \\ \operatorname{sign}(\sigma) \frac{900}{9185}, & \forall|\sigma|>\frac{900}{9185} .\end{cases}
$$

The graph of such a nonlinearity is shown in Fig. 9.

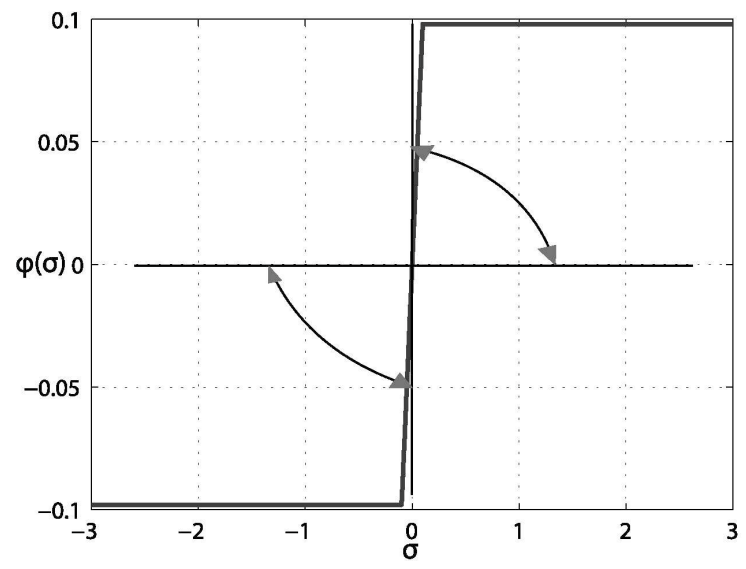

Figure 9: The graph of $\varphi(\sigma)$ and the stability sector

Modeling the solution of system (5.38) with initial data $x_{1}(0)=0, x_{2}(0)=1 / 2, x_{3}(0)=0, x_{4}(0)=0$, we obtain the periodic solution shown in Fig. 10.

To construct counterexample to Kalman's conjecture here one has to choose a nonlinearity "close" to $\operatorname{sat}(\sigma)$ and with positive derivative (because linear stability sector here is $(0, \mathrm{k})$ ). 

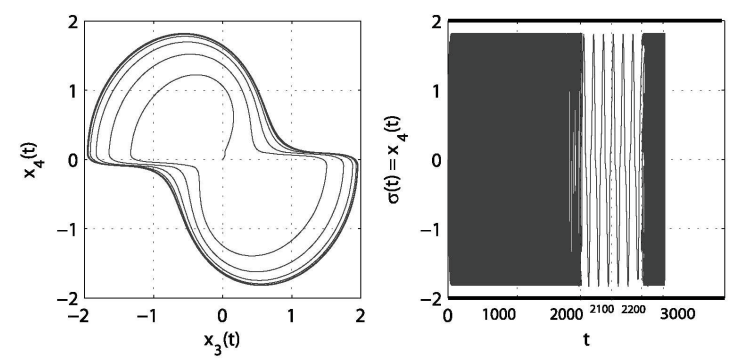

Figure 10: Trajectory of system (5.38) projection on $\left(x_{3}, x_{4}\right)$

It should be mentioned that in the examples considered above the search procedure for systems with periodic solutions is empirical. The search for systems themselves, as well as for their solutions, is very timeand labor- consuming.

In the present work an effective algorithm for the construction of classes of systems (5.31), for which Kalman's conjecture is untrue, is suggested.

\subsection{Justification of harmonic linearization in critical case}

It is well known that the method of harmonic balance gives wrong answer to Aizerman and Kalman problems (no periodic solutions: $a_{0}$ from corollary 1 is always equal to zero). But for a special class of nonlinearities there it is possible to justify describing functions method.

Let us assume first that $\mu_{1}=0, \mu_{2}>0$ and consider system $(2.5)$ with nonlinearity $\varphi^{0}(\sigma)$ of special form

$$
\varphi^{0}(\sigma)= \begin{cases}\mu \sigma, & \forall|\sigma| \leq \varepsilon \\ \operatorname{sign}(\sigma) M \varepsilon^{3}, & \forall|\sigma|>\varepsilon .\end{cases}
$$

Here $\mu<\mu_{2}$ and $M$ are certain positive numbers, $\varepsilon$ is a small positive parameter.

Then the following result occurs.

Theorem 3 (|Leonov(2009a)|) If the inequalities

$$
\begin{aligned}
& b_{1}<0, \\
& 0<\mu b_{2} \omega_{0}\left(c^{*} b+b_{1}\right)+b_{1} \omega_{0}^{2}
\end{aligned}
$$

are satisfied, then for small enough $\varepsilon$ system (2.5) with nonlinearity $\phi=\mathbf{b} \varphi^{0}$ has orbitally stable periodic solution, satisfying the following relations

$$
\begin{aligned}
& y_{1}(t)=-\sin \left(\omega_{0} t\right) x_{2}(0)+O(\varepsilon), \\
& y_{2}(t)=\cos \left(\omega_{0} t\right) x_{2}(0)+O(\varepsilon), \mathbf{y}_{3}(t)=\mathbf{O}(\varepsilon), \\
& y_{1}(0)=O\left(\varepsilon^{2}\right), \\
& y_{2}(0)=-\sqrt{\frac{\mu\left(\mu b_{2} \omega_{0}\left(c^{*} b+b_{1}\right)+b_{1} \omega_{0}^{2}\right)}{-3 \omega_{0}^{2} M b_{1}}}+O(\varepsilon), \\
& \mathbf{y}_{3}(0)=\mathbf{O}\left(\varepsilon^{2}\right) .
\end{aligned}
$$

The methods for the proof of this theorem are developed in [|Leonov et al.(1996), Leonov(2009a), Leonov(2010)|].

Based on this theorem, it is possible to apply described above multi-step procedure for the localization of hidden oscillations: initial data obtained in this theorem allow to step aside from stable zero equilibrium and to start numerical localization of possible oscillations [|Leonov(2010), Leonov et al.(2010)|,|Bragin et al., 2010|] 
For that we consider a finite sequence of piecewise-linear functions

$$
\begin{aligned}
& \varphi^{j}(\sigma)=\left\{\begin{array}{ll}
\mu \sigma, & \forall|\sigma| \leq \varepsilon_{j} ; \\
\operatorname{sign}(\sigma) M \varepsilon_{j}^{3}, & \forall|\sigma|>\varepsilon_{j} .
\end{array}, \varepsilon_{j}=\frac{j}{m} \sqrt{\frac{\mu}{M}}\right. \\
& j=1, \ldots, m .
\end{aligned}
$$

Here function $\varphi^{m}(\sigma)$ is monotone continuous piecewise-linear function $\operatorname{sat}(\sigma)$ ("saturation").

\section{Figure 11: Transformation of $\varphi^{j}(\sigma)$ into $\operatorname{sat}(\sigma)$}

We choose $m$ in such a way that the graphs of functions $\varphi^{j}$ and $\varphi^{j+1}$ are slightly distinct from each other outside small neighborhoods of points of discontinuity.

Suppose that the periodic solution $x^{m}(t)$ of system (5.31) with monotone and continuous function $\varphi^{m}(\sigma)$ ("saturation") is computed. In this case we organize a similar computational procedure for the sequence of systems

$$
\frac{d \mathbf{x}}{d t}=\mathbf{P} x+\mathbf{q} \psi^{i}\left(\mathbf{r}^{*} \mathbf{x}\right)
$$

Here $i=0, \ldots, h, \quad \psi^{0}(\sigma)=\varphi^{m}(\sigma)$ and

$$
\psi^{i}(\sigma)=\varphi^{m}(\sigma)+ \begin{cases}0, & \forall|\sigma| \leq \varepsilon_{m} \\ i\left(\sigma-\operatorname{sign}(\sigma) \varepsilon_{m}\right) N, & \forall|\sigma|>\varepsilon_{m}\end{cases}
$$

where $N$ is a certain positive parameter such that $h N<\mu_{2}$ (using the technique of small changes, it is also possible to approach other continuous monotonic increasing functions [[Leonov et al.(2010)|]]).

The finding of periodic solutions $x^{i}(t)$ of system (5.43) gives a certain counterexample to Kalman's conjecture for each $i=1, \ldots, h$.

We now give the corresponding examples.

\subsection{Counterexamples}

Example 1. Consider a system

$$
\begin{aligned}
& \dot{x}_{1}=-x_{2}-10 \varphi\left(x_{1}-10.1 x_{3}-0.1 x_{4}\right), \\
& \dot{x}_{2}=x_{1}-10.1 \varphi\left(x_{1}-10.1 x_{3}-0.1 x_{4}\right), \\
& \dot{x}_{3}=x_{4}, \\
& \dot{x}_{4}=-x_{3}-x_{4}+\varphi\left(x_{1}-10.1 x_{3}-0.1 x_{4}\right) .
\end{aligned}
$$

Here for $\varphi(\sigma)=k \sigma$ linear system (5.44) is stable for $k \in(0,9.9)$ and by the above-mentioned theorem for piecewise-continuous nonlinearity $\varphi(\sigma)=\varphi^{0}(\sigma)$ with sufficiently small $\varepsilon$ there exists periodic solution.

Now we make use of the algorithm of constructing of periodic solutions. Suppose $\mu=M=1, \varepsilon_{1}=$ $0.1, \varepsilon_{2}=0.2, \ldots, \varepsilon_{10}=1$. For $j=1, \ldots, 10$, we construct sequentially solutions of system (5.44), assuming that by $(5.42)$ the nonlinearity $\varphi(\sigma)$ is equal to $\varphi^{j}(\sigma)$. Here for all $\varepsilon_{j}, j=1, \ldots, 10$ there exists periodic solution.

At the first step for $j=0$ by the theorem the initial data of stable periodic oscillation take the form:

$$
\begin{aligned}
& x_{1}(0)=O(\varepsilon), x_{3}(0)=O(\varepsilon), x_{4}(0)=O(\varepsilon), \\
& x_{2}(0)=-1.7513+O(\varepsilon) .
\end{aligned}
$$



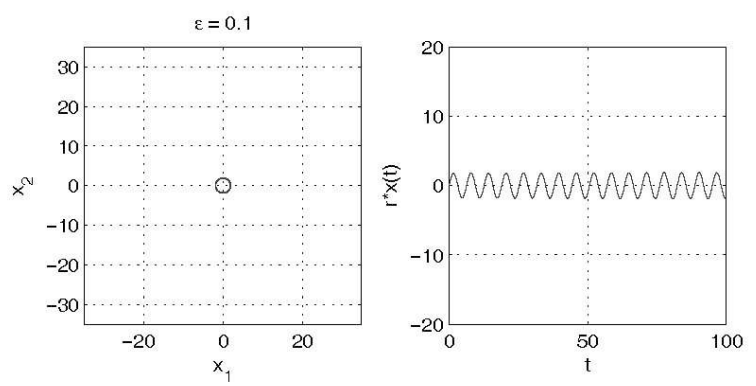

Figure 12: $\varepsilon=0.1$ : trajectory projection on the plane $\left(x_{1}, x_{2}\right)$

Therefore for $j=1$ a trajectory starts from the point $x_{1}(0)=x_{3}(0)=x_{4}(0)=0, x_{2}(0)=-1.7513$. The projection of this trajectory on the plane $\left(x_{1}, x_{2}\right)$ and the output of system $r^{*} x(t)=x_{1}(t)-10.1 x_{3}(t)-0.1 x_{4}(t)$ are shown in Fig. 12.

From the figure it follows that after transient process stable periodic solution is reached. At the first step the computational procedure is ended at the point $x_{1}(T)=0.7945, x_{2}(T)=1.7846, x_{3}(T)=0.0018, x_{4}(T)=$ -0.0002 , where $T=1000 \pi$.

Further, for $j=2$ we take the following initial data: $x_{1}(0)=0.7945, x_{2}(0)=1.7846, x_{3}(0)=0.0018, x_{4}(0)=$ -0.0002 , and obtain next periodic solutions.

Figure 13: $\varepsilon=0.2$ : trajectory projection on the plane $\left(x_{1}, x_{2}\right)$

Proceeding this procedure for $j=3, \ldots 10$, we sequentially approximate (Fig. 14.20) a periodic solution of system (5.44) (Fig. 21).

Figure 14: $\varepsilon=0.3$ : trajectory projection on the plane $\left(x_{1}, x_{2}\right)$

Figure 15: $\varepsilon=0.4$ : trajectory projection on the plane $\left(x_{1}, x_{2}\right)$

Note that for $\varepsilon_{j}=1$ the nonlinearity $\varphi^{j}(\sigma)$ is monotone. The computational process is ended at the point $x_{1}(T)=1.6193, x_{2}(T)=-29.7162, x_{3}(T)=-0.2529, x_{4}(T)=1.2179$, where $T=1000 \pi$.

We also remark that here if instead of sequential increasing of $\varepsilon_{j}$, we compute a solution with initial data according to (5.41) for $\varepsilon=1$, then the solution will "falls down" to zero.

Change the nonlinearity $\varphi(\sigma)$ to the strictly increasing function $\psi^{i}(\sigma)$, where $\mu=1, \varepsilon_{m}=1, N=0.01$, for $i=1, \ldots, 5$, and, continue the sequential construction of periodic solutions for system (5.44). The graph of such nonlinearity is shown in Fig. 22.

The periodic solutions obtained are shown in Fig. 23-27.

In the case of the computation of solution for $i=6$ there occurs the vanishing of periodic solution (Fig. 28). 

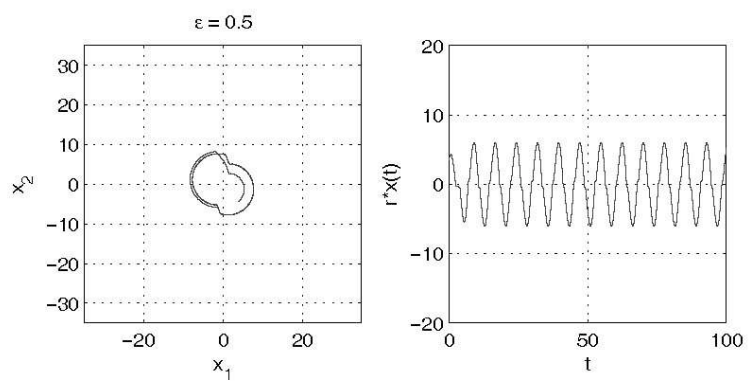

Figure 16: $\varepsilon=0.5$ : trajectory projection on the plane $\left(x_{1}, x_{2}\right)$

Figure 17: $\varepsilon=0.6$ : trajectory projection on the plane $\left(x_{1}, x_{2}\right)$

Figure 18: $\varepsilon=0.7$ : trajectory projection on the plane $\left(x_{1}, x_{2}\right)$

Figure 19: $\varepsilon=0.8$ : trajectory projection on the plane $\left(x_{1}, x_{2}\right)$

Figure 20: $\varepsilon=0.9$ : trajectory projection on the plane $\left(x_{1}, x_{2}\right)$
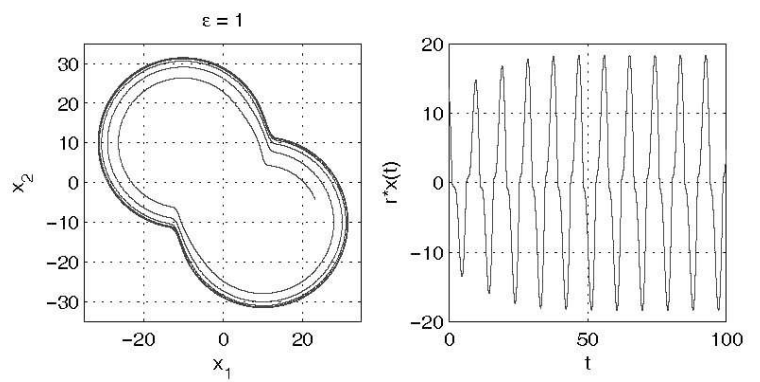

Figure 21: $\varepsilon=1$ : trajectory projection on the plane $\left(x_{1}, x_{2}\right)$

Example 2. For system (5.44) with smooth strictly increasing nonlinearity

$$
\varphi(\sigma)=\tanh (\sigma)=\frac{e^{\sigma}-e^{-\sigma}}{e^{\sigma}+e^{-\sigma}}
$$

there exists a periodic solution (Fig. 29). Here

$$
0<\frac{d}{d \sigma} \tanh (\sigma) \leq 1, \forall \sigma .
$$




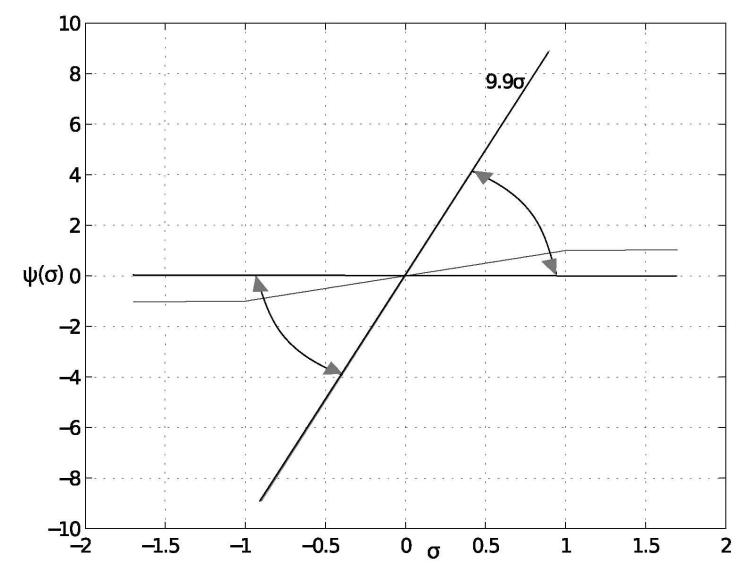

Figure 22: The graph of $\psi^{i}(\sigma)$ for $\mathrm{i}=5$ and stability sector
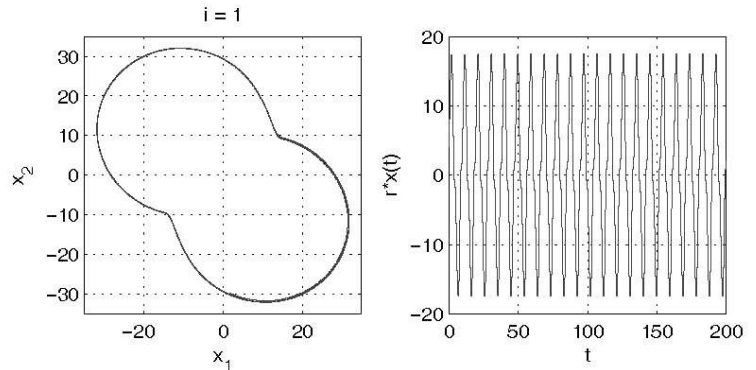

Figure 23: $\quad 1=1$ : trajectory projection on the plane $\left(x_{1}, x_{2}\right)$

Figure 24: $\quad 1=2$ : trajectory projection on the plane $\left(x_{1}, x_{2}\right)$

Figure 25: $1=3$ : trajectory projection on the plane $\left(x_{1}, x_{2}\right)$

Figure 26: $\quad 1=4$ : trajectory projection on the plane $\left(x_{1}, x_{2}\right)$

Here on the first step it is possible to apply described above method to reach saturation function; on the second - "slightly" by small steps transform saturation to tanh. 

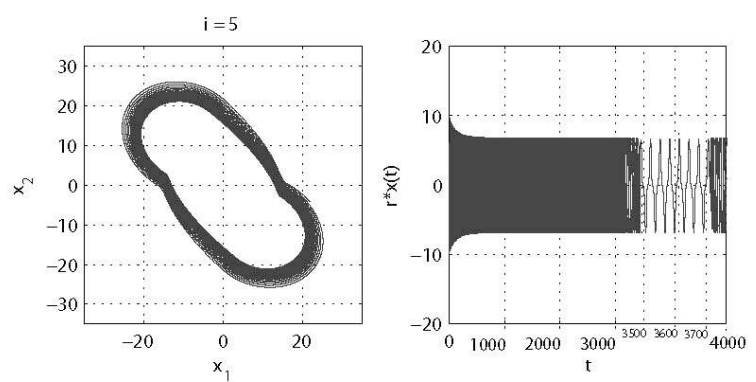

Figure 27: $1=5$ : trajectory projection on the plane $\left(x_{1}, x_{2}\right)$
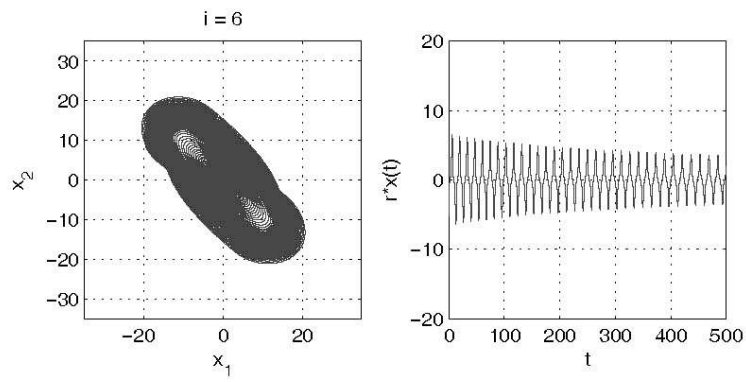

Figure 28: $\quad 1=6$ : trajectory projection on the plane $\left(x_{1}, x_{2}\right)$
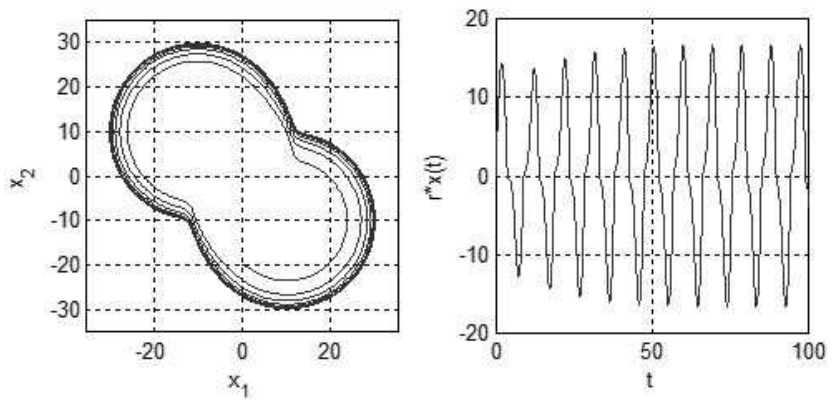

Figure 29: The projection of trajectory with the initial data $x_{1}(0)=x_{3}(0)=x_{4}(0)=0, x_{2}(0)=-20$ of system (5.45) on the plane $\left(x_{1}, x_{2}\right)$

\section{References}

[Aizerman(1949)] M.A. Aizerman. On a problem concerning the stability "in the large" of dynamical systems. Russian mathematical surveys, Vol. 4, Iss. 4, 186-188, 1949.

[Andronov et al.(1966)] A.A. Andronov, A.A. Vitt, S.E. Khaikin. Theory of Oscillators. Pergamon, Oxford, 1966.

[Barabanov(1980)] N.E. Barabanov. 1980. Meetings of the Leningrad Mathematical Society. Russian mathematical surveys, 37:1(223), 164, 1982. [in Russian]

[Barabanov(1988)] N.E. Barabanov. On the Kalman problem Sib. Math. J., 29(3), 333-341, 1988. 
[Barabanov et al.(1996)] N.E. Barabanov et al. Frequency theorem (Yakubovich-Kalman lemma) in the control theory. Avtomatika i Telemekhanika, Iss. 9, 3-40, 1996.

[Barboza \& Chua(2008)] R. Barboza, L.O. Chua. The Four-Element Chuas Circuit. International Journal of Bifurcation and Chaos, Vol. 18, No. 4, 943-955, 2008.

[Bernat \& Llibre(1996)] J. Bernat, J. Llibre. Counterexample to Kalman and Markus-Yamabe conjectures in dimension larger than 3. Dynamics of Continuous, Discrete and Impulsive Systems, Vol. 2, N3. 337-379, 1996.

[Bilotta \& Pantano(2008)] E. Bilotta, P. Pantano. A gallery of Chua attractors World scientific series on nonlinearscience. Series A. Vol. 61, 2008.

[Bragin et al., 2010] V.O. Bragin, N.V. Kuznetsov, G.A. Leonov. Algorithm for counterexamples construction for Aizerman's and Kalman's conjectures. IFAC Proceedings Volumes (IFAC-PapersOnline), 4(1). 2010. (DOI: 10.3182/20100826-3-TR-4016.00008)

[Chen \& Ueta(2002)] G. Chen, T. Ueta. Chaos in Circuits and Systems World scientific series on nonlinear science. Series B. Vol. 11, 2002.

[Chua \& Lin(1990)] L.O. Chua, G.-N. Lin. Canonical Realization of Chua's Circuit Family IEEE Transactions on Circuits and Systems, Vol. 37, No.4, 885-902, 1990.

[Chua(1992a)] L. O. Chua. The Genesis of Chua's Circuit. Archiv fur Elektronik und Ubertragungstechnik, 46, 250-257, 1992. ${ }^{a}$

[Chua(1992b)] L.O. Chua. A Zoo of Strange Attractors from the Canonical Chua's Circuits Proceedings of the 35th Midwest Symposium on Circuits and Systems, IEEE, Vol.2, 916-926, 1992. ${ }^{b}$

[Erugin(1952)] N.P. Erugin. A Problem in the theory of stability of automatic control systems. Applied Maht. \& Mech., 5, 620-628, 1952.

[Fitts(1966)] R.E. Fitts. Two counterexamples to Aizerman's conjecture Trans. IEEE., V. AC-11, N3, 553556, 1966.

[Glutsyuk(1997)] A.A. Glutsyuk. 1997. Meetings of the Moscow Mathematical Society. Russian mathematical surveys, 53:2, 413-417, 1998.

[Kalman(1957)] R.E. Kalman. Physical and Mathematical mechanisms of instability in nonlinear automatic control systems Transactions of ASME, 79:3, 553-566, 1957.

[Khalil(2002)] H.K. Khalil. Nonlinear Systems. Prentice Hall, New Jersey, 2002.

[Krasovsky(1952)] N.N. Krasovsky. Theorems on the stability of motions determined by a system of two equations. Applied Maht. \& Mech., 16(5), 547-554, 1952.

[Kuznetsov \& Leonov(2008)] N.V. Kuznetsov, G.A. Leonov. Lyapunov quantities, limit cycles and strange behavior of trajectories in two-dimensional quadratic systems. Journal of Vibroengineering, Vol. 10, Iss. 4, 460-467, 2008.

[Lefschetz(1965)] S. Lefschetz. Stability of Nonlinear Control Systems. Academic Press, NY-L, 1965.

[Leonov et al.(1995)] G.A. Leonov, D.V. Ponomarenko, V.B. Smirnova. Local instability and localization of attractors. From stochastic generator to Chua's systems. Acta Applicandae Mathematicae, Vol. 40, Iss. 3, 179-243, 1995. 
[Leonov et al.(1996)] G.A. Leonov, I.M. Burkin, A.I. Shepeljavyi. Frequency methods in oscillation theory. Kluwer Acad. Publ., 1996.

[Leonov(2006)] G.A. Leonov. Generalization of the Andronov-Vitt theorem Regular and Chaotic Dynamics, Vol. 11, Iss. 2, 281-286, 2006.

[Leonov \& Kuznetsov(2007)] G.A. Leonov: N.V. Kuznetsov. Time-Varying Linearization and the Perron effects. International Journal of Bifurcation and Chaos, Vol. 17, No.4, 1079-1107, 2007.

[Leonov(2008a)] G.A. Leonov. Strange Attractors and Classical Stability Theory. Nonlinear Dynamics and Systems Theory. Vol. 8. No. 1. 49-96, 2008.

[Leonov(2008b)] G.A. Leonov. Strange Attractors and Classical Stability Theory. St.Petersburg University Press, 2008

[Leonov(2009a)] G.A. Leonov. On Aizerman problem. Automation and remote control, No. 7, 37-49, 2009.

[Leonov(2009b)] G.A. Leonov. On harmonic linearization method. Doklady Akademii Nauk. Physcis, Vol. 424, No. 4, 462-464, 2009 ${ }^{a}$.

[Leonov(2009c)] G.A. Leonov. On harmonic linearization method. Automation and remote controle, 5, 65-75, $2009^{b}$.

[Leonov(2010)] G.A. Leonov. Effective methods for periodic oscillations search in dynamical systems. App. math. \& mech., Vol. 74, Iss. 1, 37-73, 2010.

[Leonov et al.(2010)] G.A. Leonov, V.O. Bragin, N.V. Kuznetsov. Algorithm for Constructing Counterexamples to the Kalman Problem. Doklady Mathematics, Vol. 82, No. 1, 540-542, 2010.

[Leonov et al.(2010)] G.A. Leonov, V.I. Vagaitsev, N.V. Kuznetsov. Algorithm for localizing Chua attractors based on the harmonic linearization method. Doklady Mathematics, Vol. 82, No. 1, 663-666, 2010.

[Leonov et al.(2011)] G.A. Leonov: N.V. Kuznetsov, E.V. Kudryashova. A Direct Method for Calculating Lyapunov Quantities of Two-Dimensional Dynamical Systems. Proceedings of the Steklov Institute Of Mathematics, Vol. 272, Suppl. 1, S119-S127, 2011.

[Malkin(1952)] I.G. Malkin. On the stability of automatic control systems. Applied Maht. E Mech., 16(4), 495499, 1952.

[Matsumoto(1984)] T. Matsumoto. A Chaotic Attractor from Chuas Circuit IEEE Transactions on Circuits and Systems, Vol. CAS-31. No.12, 1055-1058, 1984.

[Meisters(1996)] G. Meisters. A Biography of the Markus-Yamabe Conjecture, 1996. www.math.unl.edu/ gmeisters1/papers/HK1996.pdf

[Pliss(1958)] V.A. Pliss. Some Problems in the Theory of the Stability of Motion. Izd LGU, Leningrad, 1958.

[Savaci \& Gunel(2006)] F.A. Savaci, S. Gunel. Harmonic Balance Analysis of the Generalized Chua's Circuit. International Journal of Bifurcation and Chaos, Vol. 16, No. 8, 2325-2332, 2006.

[Stoker(1950)] J.J. Stoker. Nonlinear Vibrations in Mechanical and Electrical Systems. Interscience, NY-L, 1950. 
[Suykens et al.(1997)] J.A.K. Suykens, A. Huang, L.O. Chua. A Family of n-scroll Attractors from a Generalized Chuas Circuit AEU-International Journal of Electronics \&3 Communications, Vol. 51, No. 3, 131-138, 1997.

[Timoshenko(1928)] S. Timoshenko. Vibration Problems in Engineering. Van Nostrand, NY-L, 1928.

[Kuznetsov et al.(2010)] N.V. Kuznetsov, G.A. Leonov, V.I. Vagaitsev. Analytical-numerical method for attractor localization of generalized Chua's system. IFAC Proceedings Volumes (IFAC-PapersOnline), 4(1). 2010. (DOI: 10.3182/20100826-3-TR-4016.00009)

[van der Pol(1920)] B. van der Pol. A theory of the amplitude of free and forced triode vibrations. Radio Review, 1, 701-710, 754-762, 1920.

[van der Pol(1926)] B. van der Pol. On relaxation-oscillations. The London, Edinburgh, and Dublin Philosophical Magazine and Journal of Science, Ser. 7, 2, 978-992, 1926. 